\title{
PROXIMITY MODE INCLINED UV LITHOGRAPHY
}

\author{
Yong-Kyu Yoon and Mark G. Allen \\ School of Electrical and Computer Engineering \\ Georgia Institute of Technology \\ Atlanta, GA 30332
}

\begin{abstract}
Proximity mode inclined UV lithography is a fabrication approach enabling the single-mask realization of solid and hollow three-dimensional microstructures of unusual shapes. Expanding upon previous inclined exposure approaches, a defined gap between the photomask and the substrate adds an additional degree of freedom to generate different ray trace patterns in the photoresist layer. The proximity approach can be used with both front-side and reverse-side exposure approaches: the air gap is controlled by spacers with different thicknesses between the photomask and the substrate for front-side exposure, while UV transparent glass of known thickness on the substrate prior to photoresist deposition enables proximity reverse-side exposure. With continuously varying air gap spacing, nozzles with various orifice sizes of $0 \mu \mathrm{m}$ to $255 \mu \mathrm{m}$, a height of $250 \mu \mathrm{m}$, a side wall tilting angle of $25^{\circ}$, a wall thickness of approximately $60 \mu \mathrm{m}$ have been successfully fabricated using front-side exposure with an incident angle of $45^{\circ}$ and $50 \mu \mathrm{m}$-diameter of clear circle mask patterns. A horn shape has been achieved by reverse-side inclined exposure using the same photomask patterns used in nozzle fabrication.
\end{abstract}

\section{INTRODUCTION}

Placement of photomasks in proximity to, rather than in contact with, the substrate has been widely used in standard UV lithography to prevent contamination or damage of the mask or the substrate $[\mathbf{1 , 2}]$ and for photoresist patterning on an uneven substrate [3]. In conventional proximity patterning, since the UV source is incident normal to the substrate, the transferred patterns follow the photomask image in shape, potentially with reduced resolution due to light diffraction at the edge of the pattern.

Recently, advanced UV lithography processes using SU-8 such as inclined exposure and reverse-side exposure have been reported for complex three-dimensional (3-D) fabrication [4]. When the inclined exposure technique is utilized in a rotational fashion, e.g. the substrate stage rotating during exposure, various patterns of revolution can be produced.

In this paper, the inclined rotational exposure process has been further advanced by exploiting the proximity scheme to generate unusual 3-D patterns, which are different from the original mask patterns. Since the proximity gap between the mask and the photoresist plays an essential role to determine the resultant 3-D image, the gap effects for 3-D patterning have been investigated. To demonstrate its versatility, tapered micronozzles and conical microhorns have been fabricated from front-side exposure and reverse-side exposure, respectively. Mathematical equations for resultant dimension as a function of gap and incident angle have been provided and compared with the fabricated results.

\section{PROXIMITY MODE INCLINED UV LITHOGRAPHY}

Two types of proximity modes are illustrated in Figure 1. Figure 1a shows an air gap inserted between the mask and the substrate for front-side exposure. The gap can be controlled by placing spacers of known thickness. A ray trace through a clear window with a diameter of $d_{m}$ in the optical mask can generate a 3D nozzle latent pattern of revolution in the SU-8 layer after inclined rotational exposure, while its geometrical dimensions can be determined by the incident angle $\theta_{i}$, the refractive index of SU$8 n_{S U-8}(\cong 1.67[4])$, the thickness of the photoresist layer $t$, and the gap between the photomask and the substrate $g$, and are described as follows:

$d_{\text {oti }}:$ inner diameter of orifice tip $=2 * g / \tan \theta_{i}-d_{m}$

$d_{\text {oto }}$ : outer diameter of orifice tip $=2 * g / \tan \theta_{i}+d_{m}$

$d_{\text {ori }}$ : inner diameter of orifice root $=2 * g / \tan \theta_{i}-d_{m}+2 * t * \tan \theta_{r}$. (3)

$d_{\text {oro }}$ : outer diameter of orifice root $=2 * g / \tan \theta_{i}+d_{m}+2 * t * \tan \theta_{r}$ (4)

$\theta_{r}:$ refracted angle $=\sin ^{-1}\left(\sin \theta_{i} * n_{\text {air }} / n_{S U-8}\right)$

Proximity patterning can be implemented for reverse-side exposure by adding a known-thickness gap layer prior to SU-8 deposition as shown in Figure 1b, where both the substrate and the gap layer are UV transparent, e.g. glass. However, the gap layer is not limited to glass but can be UV-transparent polymer or ceramic. The substrate has a prepatterned metal layer for a photomask, having a clear open window. The resultant pattern after reverseside inclined rotational exposure will produce a horn shape.

Figure 2 shows structures fabricated from the same photomask with $50 \mu \mathrm{m}$ diameter clear window patterns: (a) a nozzle from front-side exposure, (b) a horn array with the lower portion truncated by the gap layer from reverse-side exposure, (c) a horn with a central column fabricated from additional vertical exposure after reverse-side inclined rotational exposure using the same mask.

As a reference, the contact mode structures are shown in Figure 3: (a) a closed top conical shape from front exposure, (b) a horn from reverse-side exposure, and (c) a multi-layer horn with various exposure angles from reverse-side exposure. The tips of the microcones or microhorns are the same size as the mask pattern.

\section{MICRONOZZLE ARRAY}

By implementing continuously varying air gaps between the photomask and the substrate, a micronozzle array with different orifice sizes can be simultaneously formed as shown in Figure 4. The gap of the leftmost side is set to zero and that of the rightmost side has a spacer with a thickness of approximately $500 \mu \mathrm{m}$. The gap $g$ is a function of the distance $x$ from the leftmost side. Since the mask width is 1 " $(=25.4 \mathrm{~mm})$, the gap is described as a function of the distance:

$$
g=0.5 x / 25.4 \cong 0.02 x
$$


The mask tilting angle $\theta_{m}$ is approximately $2^{\circ}$ and therefore the overall nozzle shape is not noticeably asymmetric due to the tilting gap. Figure 5 shows a fabricated micronozzle array with various orifice sizes. The inner and outer orifice diameters of the fabricated nozzles are calculated using Eq. (1) and (2), respectively and compared with the measured ones as shown in Figure 6. In the calculation, the nozzle width has been assumed to be the same as the original opening diameter $d_{m}(50 \mu \mathrm{m})$ in the mask. Taking into account diffraction through the air gap, the resultant wall thickness could be widened as much as $1 \sim 30 \mu \mathrm{m}$ in the air gap range utilized [1], showing good agreement with the measurement results.

\section{CONCLUSION}

We demonstrate that incorporated with inclined rotational UV exposure, proximity lithography could produce solid and hollow structures of revolution such as a nozzle or horn structure from a single mask pattern. The proximity approach provides an additional process parameter, i.e. gap, in addition to inclined angle, refractive index of photoresist, and thickness of the photoresist of inclined rotational exposure, for the fabrication of 3-D microstructures. Both front-side and reverse-side exposure could exploit the gap effects by changing the air gap between the photomask and the substrate or by adding a gap layer between the mask layer and the photoresist. A nozzle array with various orifice sizes has been simultaneously obtained using a continuously varying gap. The fabricated micronozzles or microhorns may have potential for microfluidic, biomedical, or RF antenna applications.

\section{ACKNOWLEDGEMENT}

The authors would like to thank Mr. Seong-O Choi, Dr. Jung-Hwan Park, and Dr. Mark Prausnitz at Georgia Tech for their valuable discussion.

\section{REFERENCES}

1. C.G. Wilson, "Organic Resist Materials," in Introduction to Microlithography, L.F. Thompson, C.G. Wilson, and M.J. Bowden, Eds. Washington D.C.; American Chemical Society, 1994.

2. W. Henke, M. Weiss, R. Schwalm, and J. Pelka, "Simulation of proximity printing," Microelectron. Eng. vol. 10, pp 127-52, 1990.

3. Y.-K. Yoon, M.G. Allen, et al. "Surface Micromachined Electromagnetically Radiating RF MEMS," Solid State Sensor, Actuator, and Microsystems Workshop 2004, Hilton Head Island SC, pp. 328-331.

4. Y.-K. Yoon, J.-H. Park, and M.G. Allen, "Multidirectional UV lithography for complex 3-D MEMS Structures," IEEE Journal of MEMS (in press)

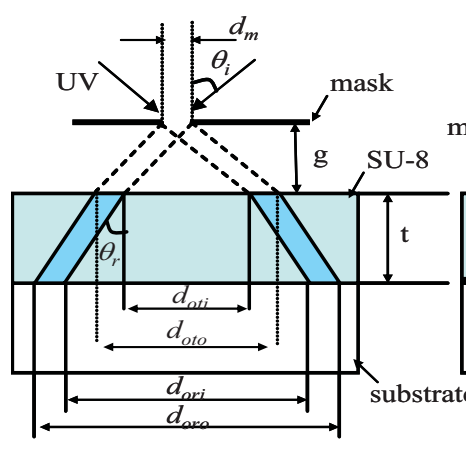

(a)

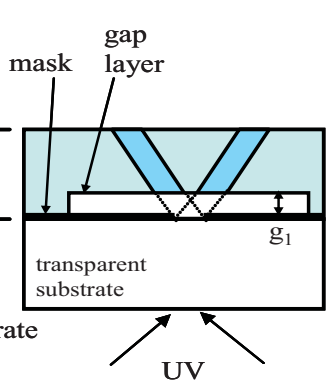

(b)
Figure 1. Proximity mode inclined UV lithography: (a) front-side exposure, (b) reverse-side exposure through a transparent substrate and a gap layer.

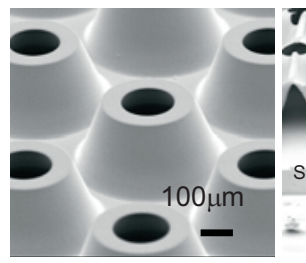

(a)

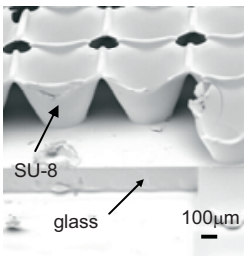

(b)

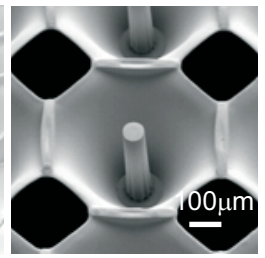

(c)
Figure 2. Proximity mode inclined rotational UV lithography: (a) front-side exposure, (b) reverse-side exposure with a $200 \mu \mathrm{m}$ thick glass gap, and (c) reverse-side exposure with a $200 \mu \mathrm{m}$ glass gap and an additional vertical exposure for the central column

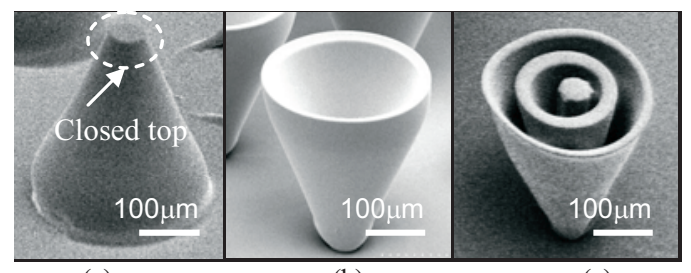

(a)

(b)

(c)

Figure 3. Contact mode inclined rotational UV lithography: (a) frontside exposure, (b) reverse-side exposure, and (c) reverse-side exposure with multiple inclined angles

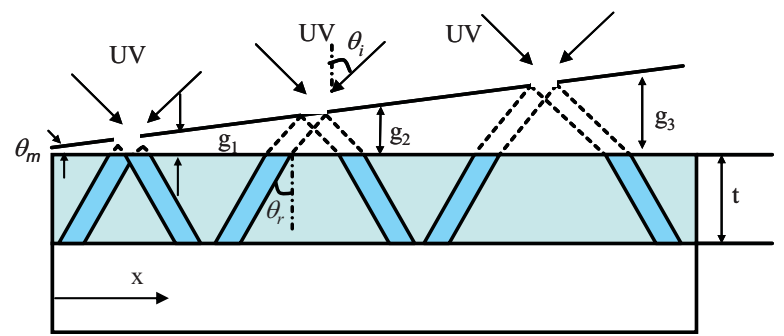

Figure 4. Nozzles fabricated using proximity mode inclined rotational exposure with a continuously varying gap

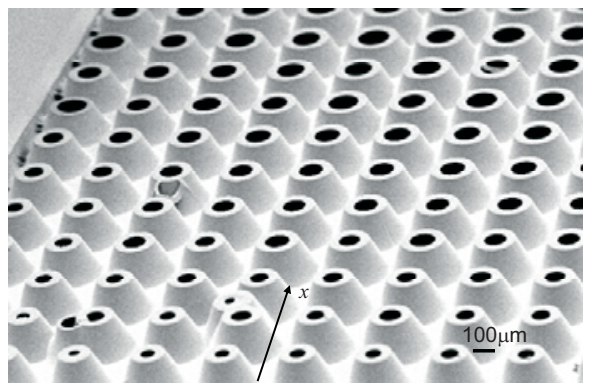

Figure 5. Fabricated micronozzle array with various orifice sizes resulting from continuously varying gap

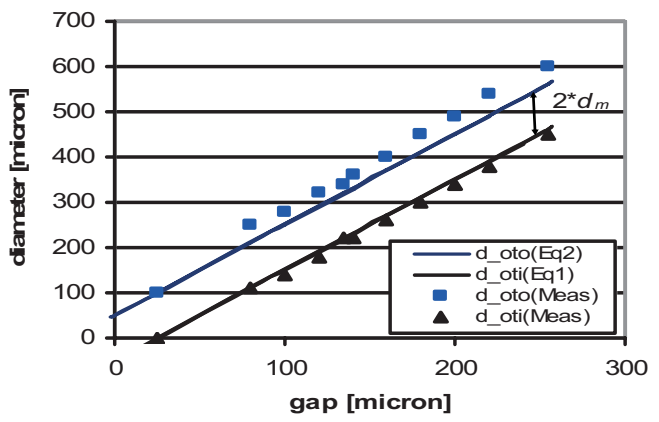

Figure 6. Outer diameter of orifice tip $\left(d_{\text {oto }}\right)$ and inner diameter $\left(d_{\text {oti }}\right)$ as a function of gap 